\title{
ERROS MÉDICOS NAS PRESCRIÇÕES DE MEDICAÇÕES
}

\section{ARTIGO DE REVISÃO}

SAVIAN, Tiago Rezende ${ }^{1}$

FRAGA, Carolina Cândida De Resende ${ }^{2}$

FRAGA, Ana Laísa Cândida De Resende ${ }^{3}$

SAVIAN, Tiago Rezende. FRAGA, Carolina Cândida De Resende. FRAGA, Ana Laísa Cândida De Resende. Erros médicos nas prescrições de medicações. Revista Científica Multidisciplinar Núcleo do Conhecimento. Ano 05, Ed. 01, Vol. 04, pp. 4661. Janeiro de 2020. ISSN: 2448-0959, Link de acesso: https://www.nucleodoconhecimento.com.br/saude/erros-medicos

\section{RESUMO}

O erro médico ocorre quando um profissional de saúde escolhe um método inadequado de cuidado ou, de forma inadequada executa um método correto. São frequentemente descritos como erros humanos na área da saúde. No entanto, as definições de erro médico estão sujeitas a debate, pois existem muitos tipos de erros médicos, de menor para maior gravidade, e a causalidade é muitas vezes mal

\footnotetext{
${ }^{1}$ Médico Generalista graduado pela UNIMES - Universidade Metropolitana de Santos, cidade de Santos/SP. Advogado e bacharel em direito graduado pelo CESUR - Centro de Ensino Superior de Rondonópolis, cidade de Rondonópolis/MT. Pós-graduado em Direito Processual do Trabalho pela UGF - Universidade Gama Filho.

2 Fisioterapeuta graduada pela PUC-GO - Pontifícia Universidade Católica de Goiás, cidade de Goiânia/GO. Acadêmica do $6^{\circ}$ ano do curso de medicina da UNIMES Universidade Metropolitana de Santos.

${ }^{3}$ Médica Veterinária graduada e Mestre pela UFMT - Universidade Federal do Mato Grosso, cidade de Cuiabá/MT. Acadêmica do $3^{\circ}$ ano do curso de medicina da FAMP - Faculdade Morgana Potrich, cidade de Mineiros/GO.
} 
determinada. O objetivo deste estudo de revisão foi desmistificar os erros médicos, com foco primordial na prescrição medicamentosa, identificando os erros e riscos que o cidadão/paciente pode ser acometido levando em consideração a falta de legibilidade, e o que pode ser feito para minimizar esses erros. Concluiu-se, do ponto de vista ético, que ambas as áreas estão cometendo erros, seja na prescrição das receitas ou durante a interpretação, sendo indispensável a fiscalização de todas as prescrições médicas.

Palavras-chaves: Prescrições médicas, erros médicos, erros de medicação.

\section{INTRODUÇÃO}

As receitas médicas muitas vezes são alvo de especulações, mas nada que possa ser levada a alguma mudança em benefício da sociedade. O termo "letra de médico" é um antigo jargão, e pelo visto atual. Em um passado, nem tão remoto, as famílias tinham seus médicos familiares que iam até a casa prescrever tratamento ao paciente, e este não se preocupava em saber o que o médico havia prescrito. Mas hoje, com todo o avanço tecnológico e de informação virtual, esta preocupação está sendo cogitada, pois sua saúde e bem-estar depende da administração correta do medicamento, já que o paciente necessita dele conforme andamento da doença.

Este estudo tentará mostrar os dois lados em discussão neste artigo, analisando tanto o trabalho dos médicos como também a responsabilidade do estado, em benefício à sociedade. A metodologia de pesquisa é teórica bibliográfica, descritiva com base em bibliografia encontrada em sites dos Conselhos regionais de medicina, e conselhos regionais de farmácia, artigos sobre o tema, Jurisprudência e livros em bibliotecas virtuais.

Para melhor entender sobre prescrições médicas, deve-se considerar alguns fatores que possam prejudicar a caligrafia, e a produção destas receitas; pressa, urgência, sobrecarga de trabalho, estresse, dupla jornada, poucos médicos e muitos pacientes, principalmente, se atendem em órgãos públicos, onde existe uma maior cobrança 
para que os mesmos atendam com maior agilidade, devido à alta demanda de pacientes[4].

Sabe-se que é fato: o profissional da área da saúde tem dificuldade em lidar com o erro humano, pois são ensinados que estão acolhendo vidas e os erros são inaceitáveis. Artigos relacionados ${ }^{1,}$ [5] retratam como erro identificado a falta de legibilidade nas prescrições. Ocorrem vários outros erros, que não são o foco da presente pesquisa, mas serão descritos durante as explanações, já que geralmente são provenientes da mesma pressa demasiada que ocasiona a ilegibilidade.

Nesta revisão bibliográfica foram analisados em um primeiro momento o que são erros médicos de uma forma mais ampla, e foram sugeridas e recomendadas soluções, para prevenção e correção, discutindo providências que podem e já estão sendo implantadas no sistema de saúde público e no privado.

\section{A QUANTIFICAÇÃO DO PROBLEMA}

De acordo com Zhang, Patel e Johnson[6] um erro médico ocorre quando um profissional de saúde escolhe um método inadequado de cuidado ou, de forma inadequada executa um método correto. Os erros médicos são frequentemente descritos como erros humanos na área da saúde. No entanto, as definições de erro médico estão sujeitas a debate[7], pois existem muitos tipos de erro médico, de menor para maior gravidade, e a causalidade é muitas vezes mal determinada.

Um relatório do Institute of Medicine (IM) de 2000 escrito por Donaldson et al.[8] estimou que os erros médicos são cerca de 40 mil, e, são 98 mil mortes evitáveis e 1.000.000 de indivíduos com ferimentos graves e injúrias decorrentes dos erros, nos hospitais dos Estados Unidos da América (EUA).

Alguns pesquisadores, como Hayward et al.[9] questionaram a veracidade do estudo do IM, criticando o tratamento estatístico dos erros de medição no relatório, a subjetividade significativa na determinação de que as mortes eram "evitáveis" ou 
devido a erro médico, e uma suposição errônea de que 100\% dos pacientes teriam sobrevivido se os melhores cuidados fossem prestados.

Um estudo de 2001 publicado no Journal of the American Medical Association estima que para cada 10.000 pacientes internados em hospitais, um paciente morre, e este teria gozado até três meses ou mais de boa saúde cognitiva se o cuidado fornecido fosse classificado como "ótimo"[10].

Em 2006, outro estudo da IM descobriu que os erros de leitura da prescrição ou interpretação da medicação estão entre os erros médicos mais comuns, prejudicando pelo menos 1,5 milhões de pessoas a cada ano. De acordo com o estudo, 400 mil acidentes evitáveis na administração de drogas ocorrem a cada ano em hospitais, 800.000 em ambientes de cuidados de longo prazo (não sendo UTI), e cerca de 530 mil entre os beneficiários de plano médico em ambulatórios. $\mathrm{O}$ relatório afirma que estas são suscetíveis de serem estimativas conservadoras.

Só em 2000, os custos médicos adicionais incorridos por lesões evitáveis relacionadas com as drogas administradas, aproximados 887 milhões dólares - e o estudo analisou apenas ferimentos sofridos por beneficiários do Medicare (plano de saúde norteamericano), um subconjunto de visitantes da clínica. Nenhum desses valores considera os salários, a produtividade perdida ou outros custos.

De acordo com o relatório de qualidade da Agência de Investigação de Saúde (2002), cerca de 7.000 pessoas morrem anualmente devido aos erros de medicação - cerca de 16 por cento a mais de mortes do que o número atribuído a lesões relacionadas com acidentes de trabalho $(6.000 \text { mortes })^{6}$.

Os erros médicos afetam um em cada 10 pacientes no mundo inteiro. Uma extrapolação sugere que 180.000 pessoas morrem a cada ano, em parte como resultado da lesão iatrogênica. Um em cada cinco norte-americanos (22\%), relataram que eles, ou um membro da família, tem experimentado um erro médico de algum tipo 6 . 


\section{POR QUE OS ERROS OCORREM?}

Tecnologias complicadas, drogas poderosas, cuidados intensivos, e internação prolongada podem contribuir para erros médicos. Em 2000, Donaldson et al. ${ }^{5}$ afirmaram que o problema dos erros médicos não são pessoas ruins no atendimento de saúde, mas sim que as pessoas boas estão trabalhando em sistemas ruins que precisam ser mais seguros.

As linhas de comunicação e pouco claras e deficitárias entre médicos, enfermeiros e outros prestadores de cuidados também são fatores contribuintes. Os sistemas de comunicação desconectados dentro de um hospital podem resultar em sistemas fragmentados que termina em numerosas falhas nos resultados dos pacientes[11].

Outros fatores incluem a impressão de que a ação está sendo realizada por outros grupos dentro da instituição, a dependência de sistemas automatizados para evitar erro, e sistemas inadequados para compartilhar informações sobre os erros, o que dificulta a análise das causas contributivas e estratégias de melhoria. As medidas preventivas em hospitais, em resposta aos cortes de custos podem comprometer a segurança do paciente. Em caso de emergência, o pronto atendimento ao paciente pode ser prestado em áreas pouco adequadas para um monitoramento seguro[12].

O The National Academy of Science identificou preocupações para o projeto de segurança e construção de instalações de cuidados de saúde. As falhas de infraestrutura também são uma preocupação. Segundo a OMS, 50\% dos equipamentos médicos nos países em desenvolvimento são apenas parcialmente utilizáveis devido à falta de operadores qualificados ou peças faltantes. Como resultado, os procedimentos de diagnóstico ou de tratamento não podem ser realizados, levando a um tratamento de baixa qualidade[13].

De acordo com Hayward et al. ${ }^{6}$ o The Joint Commission's Annual Report on Quality and Safety, constatou que a comunicação inadequada entre os profissionais de saúde, ou entre prestadores, e os pacientes e familiares, foi a causa de mais de metade dos eventos graves adversos em hospitais credenciados. Entre outras causas principais 
incluíram avaliação inadequada da condição do paciente, e uma liderança ou formação fraca.

\section{OS ERROS E AS MEDIDAS JUDICIAIS}

A prescrição de medicamentos tem papel crucial na definição das características da demanda judicial. Os profissionais médicos são responsáveis por grande parte dos gastos em saúde por meio de sua decisão diagnóstica e/ou terapêutica. Além disso, a prescrição racional é uma das bases do uso racional e da adesão aos medicamentos, interferindo no resultado do tratamento. A prescrição é influenciada pelas condições físicas do atendimento e características do profissional de saúde, como conhecimento, perícia, formação profissional, e sua atualização sobre eficácia e segurança de antigos e novos medicamentos[14].

Em um estudo dirigido a explicitar o problema com o erro médico, referenciando a ilegibilidade de prescrições Sant'ana et al.[15] analisaram os elementos processuais, médico científico e sanitário das demandas sob as principais variáveis. Entre estas variáveis constavam a presença de prescrição médica, adequação da prescrição médica às boas práticas de prescrição, dentre outros que não cabem ao tema principal deste trabalho.

A adequação da prescrição médica aos preceitos gerais e legais de boas práticas de prescrição baseou-se em 14 critérios mínimos de normas federais - Leis Federais no 5991/73b e n 9787/99. c Portaria no SVS/MS 344/98d e Resolução CFF no 357/2001. Onde foram considerados como "de acordo" apenas quando o critério era respeitado, para caso aplicável, e para todos os medicamentos prescritos ${ }^{12}$.

No estudo de Sant'Ana et al. ${ }^{12}$ nenhuma prescrição mostrou-se adequada aos preceitos gerais e legais de boas práticas de prescrição, sendo encontrada uma mediana de cinco critérios em desconformidade por prescrição. Ainda citam que houve o predomínio de prescrições originadas no SUS (Sistema Único de Saúde). O mais preocupante é o fato de que nenhuma das prescrições analisadas tenha respeitado todos os preceitos de boas práticas de prescrição selecionados e que, da 
mesma forma, tenham sido deferidas e utilizadas como conteúdo de ações movidas contra o Estado, pelo Poder Judiciário sem exigência de adequação às leis sanitárias vigentes.

Catanheide, Lisboa e Souza[16] também fazem referência que erros em prescrições podem levar a uma série de problemas relacionados ao uso de medicamentos, como, por exemplo, troca do medicamento e/ou substituição das formas farmacêuticas na dispensação, administração do medicamento pela via errada, na dose errada, uso além ou aquém do tempo necessário ou não adesão ao tratamento.

E desta forma considerando que o foco das demandas judiciais de medicamentos, na perspectiva jurídica, é a saúde do paciente litigante, seria importante que a apreciação do Judiciário exigisse ao menos o cumprimento dos requisitos mínimos para uma prescrição segura e adequada, dos quais grande parte trata-se de exigências legais ${ }^{12,}$ 13.

Outro fato que se destaca é o baixo percentual de medicamentos prescritos pelo nome genérico no âmbito SUS, uma vez que a Lei Federal n.ำ 9.787/1999 estabelece a obrigatoriedade da adoção da denominação genérica para as prescrições médicas e odontológicas de medicamentos. Percebemos que com a grafia ilegível de alguns profissionais da área médica e odontológica, erros irreparáveis podem ser atentados ao paciente. Assim, o defensor público pode vir a fazer pedido de um medicamento errado devido a não legibilidade, assim como o paciente pode ingerir um medicamento errado pela falha na comunicação, o que não pode ocorrer, até pela proposta da ética profissional médica ${ }^{12}$.

Desta forma, por não terem condições de arcar com despesas de consultas, remédios e exames, o indivíduo hipossuficiente tem recorrido à Defensoria Pública para obter a tutela do Estado no tratamento médico. Na maioria dos casos, segundo os Defensores, ao propor uma ação perante o Estado em defesa dos direitos do paciente na justiça, acontece de um documento ser negado pela "letra ilegível dos médicos", não conseguindo, assim, saber qual o tratamento e medicamento adequado a ser requerido na ação[17]. 


\section{A SOLUÇÃO}

Existem dados da experiência hospitalar nos EUA retirados de relatos espontâneos sobre erros com medicações que demonstram que $31 \%$ estavam relacionados à equipe de enfermagem, $24 \%$ a farmácia e $13 \%$ a equipe médica, sendo que o incidente mais comum envolvia o uso do medicamento errado devido à confusão provocada pela semelhança entre embalagens, além de informações incompletas, lapsos de memória, falta de conhecimento especifico e prescrição incompleta[18].

Anacleto et al.[19] ponderam que o papel fundamental fica a cargo do farmacêutico hospitalar no desenvolvimento e na execução de processos que possam prevenir erros na dispensação das medicações. Portanto, ele representa importância essencial em todas as etapas de processo que envolve o medicamento dentro da instituição hospitalar. A sua inserção no cuidado ao paciente, junto à equipe multiprofissional, também é muito importante, apesar de ser uma abordagem relativamente recente. Ainda relatam que existe um impacto muito positivo na redução dos erros, na prevenção, nas orientações sobre as medicações e nos custos do tratamento do paciente quando há inserção do farmacêutico clínico na equipe de saúde.

Quanto à prescrição, são vários erros relatados, como visto anteriormente. Acontecem por exemplo a escolha incorreta do medicamento (sem checar as contraindicações e fatores relacionados a ocorrências de alergias), doses inadequadas, via de administração e velocidade de infusão erradas, além das prescrições ilegíveis ${ }^{16}$. Não sendo o propósito desse estudo, mas, entre os demais erros que contribuem para essa triste estatística, estão os horários de administração, as doses, a transcrição da prescrição, e na dispensação do medicamento que pode ocorrer no momento da separação do medicamento, no processo de preparo da prescrição pela farmácia.

Não se pode descartar os fatores estressantes da profissão médica, que podem levar a erros. Mas também se sabe que algumas estratégias podem diminuir os erros com os medicamentos[20]: 
- A padronização dos processos e efetiva atuação da comissão de farmácia e terapêutica (CFT);

- Protocolos devem ser por escrito, e deve se ter uma lista de checagem das rotinas e procedimento;

- Devem-se simplificar os procedimentos e processos de rotina;

- Deve haver treinamentos constantes, aulas de reciclagem e cursos e acesso de toda equipe a essas informações;

- O profissional não deve confiar apenas na memória;

- Devem-se reduzir mudanças e improvisos nos turnos de trabalho;

- Deve-se estimular a automatização de todos os processos, com a equipe, (principalmente os prescritores).

Aqui, o farmacêutico é fundamental no processo de minimização de erros ao paciente, uma vez que suas funções interferem tanto no aspecto administrativo quanto no clínico, sendo peça chave na orientação da estruturação de todos os processos que possam melhorar os serviços. Um exemplo é a implantação de serviço de informação automatizada sobre os medicamentos, a prescrição informatizada (eletrônica) e dispensação de medicamentos por características setoriais (pediatria, UTI, cirúrgica, pós-cirúrgica etc.)[21].

\subsection{INFORMATIZAÇÃO NA ÁREA HOSPITALAR}

Atualmente, os sistemas de informatização são utilizados com pouca frequência nos processos hospitalares no Brasil. Existe um grande impacto positivo na qualidade dos serviços de dispensação quando estes possuem os processos de utilização de medicamentos informatizados. Portanto a informatização é uma ferramenta que deve ser utilizada para a redução de distúrbios e eventos adversos provenientes de erros, embora sejam poucos os hospitais, no Brasil, que já aplicam essa tecnologia[22].

O sistema de identificação por código de barras para medicamentos, materiais médicos hospitalares e pacientes, apesar de ter um custo alto, pode reduzir erros com medicações, podendo adicionar informações de estrema importância, como a entrada do medicamento no hospital, o controle das validades e dos lotes, além da 
dispensação dos medicamentos e da administração, essa é toda a parte logística, e envolve também a ala da enfermagem[23].

O mercado nacional apresenta muita informação, são aproximadamente mil e quinhentos fármacos e mais de seis mil marcas, que correspondem a quinze mil apresentações comerciais. Assim é dependente do banco de dados informatizado, para que os profissionais não cometam erros relacionados com os medicamentos[24].

\subsection{PRESCRIÇÃO MÉDICA ELETRÔNICA}

Essa prescrição nada mais é do que uma programação, com um banco de dados que permite à equipe médica prescrever todas as suas decisões clínicas e medicamentosas nos arquivos do computador, substituindo o papel. A vantagem nesse sistema é que as prescrições são padronizadas, completas e legíveis, diminuindo assim, os erros provenientes delas. Muitos destes sistemas oferecem apoio de prognostico e diagnóstico em banco de dados para auxiliar na decisão clínica e terapêutica[25].

Portanto é notório que a prescrição médica eletrônica melhora a qualidade dos processos com medicamentos, modernizando e simplificando os sistemas convencionais, que geralmente envolvem muitas pessoas, registros manuais e atrasos em muitas tarefas e praticamente anula o erro pela ilegibilidade devido a caligrafia médica.

Entre as vantagens do Sistema Informatizado de Prescrição eletrônica estão[26]: melhor segurança com os dados sobre os medicamentos; facilidade ao prescrito de "cruzar" informações entre os medicamentos a serem prescritos (doses, interações, posologias etc.).; acesso à padronização de medicamentos, dietas, nutrições parenterais, protocolos quimioterápicos, hemoderivados, além de padronização de formulários e rotinas a serem seguidas; diminuição das incompatibilidades de medicamentos aos pacientes; rapidez e simplificação do processo de prescrição; redução dos custos das medicações; facilidade ao prescritor de cruzar informações 
sobre medicamentos a serem prescritos com exames radiológicos e laboratoriais; legibilidade da prescrição.

Facilidade de armazenar os dados dos prontuários de cada paciente; diminuição de erros relacionados e medicamentos; melhora da qualidade das prescrições; redução do tempo gasto com transcrição de prescrições, digitação de requisições; maior integração dos processos exigidos pelo hospital com os profissionais envolvidos; facilidade do acompanhamento e da intervenção no uso de antimicrobianos; possibilidade de estabelecer estatísticas do uso de medicamentos por classe terapêutica, especialidade médica, paciente, consumo, entre outras; possibilidade de estabelecer auditorias em todas as fases do processo da prescrição; maior controle no registro de medicamentos sob controle especial; melhor integração entre os profissionais envolvidos, evitando o desgaste; melhor terapêutica aos pacientes, diminuindo riscos à saúde deles; redução de erros e consequentemente de custos com medicamentos e processos hospitalares, melhorando a qualidade da atenção à saúde 23 .

Apesar das diversas vantagens que a implementação de um sistema informatizado de prescrição médica eletrônica traz à instituição hospitalar e consequentemente à saúde dos pacientes, não podemos deixar de considerar as dificuldades dessa execução referentes à: falta de capacitação dos profissionais na área de informática; situação financeira instável dos setores de saúde; falta de rigor e padronização dos processos, que muitas vezes são indefinidos nos hospitais, levando a dificuldade de serem informatizados; rejeição pela equipe de saúde da inovação de processos e da adequação a novos critérios por meio de educação continuada ${ }^{23}$.

As sugestões para o melhor desenvolvimento e a implantação da prescrição médica eletrônica, partem do princípio de que deve haver um bom projeto para execução, equipe multidisciplinar de saúde envolvida, e sempre é avaliado um software específico para a instituição hospitalar. Deve-se gradativamente implantar por ala, para melhor adaptação do pessoal. A educação e integração de pessoal devem ser continuadas, com treinamento e suporte técnico aos usuários do hospital. Deve haver a execução setorial, assim facilitando a correção específica[27]. 


\subsection{PADRONIZAÇÃO}

Um sistema de distribuição de medicamentos deve ser racional, eficiente, econômico, seguro e deve estar de acordo com o protocolo terapêutico descrito. Quanto mais eficiente o sistema de distribuição, maximiza a garantia do sucesso do tratamento e da profilaxia instauradas. Os aspectos que devem ser seguidos como critérios são o administrativo e econômico. Quanto à administração, deve-se gerir corretamente para ter racionalização e eficiência na distribuição. É essencial que o setor de compras esteja envolvido em tal processo. Como controle logístico são aspectos importantes: o controle do estoque, a padronização, o envolvimento de recursos humanos treinados e capacitados para as funções e os controles de qualidade de todos os procedimentos. É de extrema importância que todas as áreas sejam atendidas[28].

Já no aspecto econômico o responsável farmacêutico deve estar atento às condições econômicas vigentes no país, pois a instituição sofre interferências tanto da política econômica como de sua própria economia, deve haver a preocupação com custos e receita[29]. Existe ainda a cultura de que dois funcionários pouco especializados e experientes (mais baratos) são melhores que um profissional pleno (mais caro). Atualmente tem-se percebido que a melhor opção para economia é exatamente o contrário, pois os danos provocados por incompetência causam gastos bem mais elevados, podendo gerar perigo à vida de pacientes.

Existem alguns requisitos primordiais e necessários para implantação de um sistema de dispensação de medicamentos, que devem ser analisados em conjunto para garantir o sucesso da execução de um software. Considera-se o tipo de hospital se público ou privado; se o serviço prestado é geral ou especializado; e quais são os recursos disponíveis (humanos materiais e econômicos).

Escopo de projeto e o projeto em si é identificado melhor quando escrito e documentado, e que seja discutido e melhorado conforme as adaptações do hospital. Lembrando que cada instituição possui suas características singulares, e o farmacêutico atuante deve desenvolver o projeto considerando a realidade política e econômica. Quanto maior o planejamento menor a margem de erros após execução 
de projeto, assim é preferível gastar mais tempo planejando e analisando os pontos fracos e fortes da instituição[30].

\subsection{A CAPACITAÇÃO DE PESSOAL}

Os resultados de implantação e execução sugeridos pela diretoria do hospital, para as chefias médicas, de enfermagem e para os prescritores, devem ser feitas. E a partir do momento de sensibilização e mobilização deve ser instituído um ciclo de palestras, na intenção de atingir os profissionais dos demais setores do hospital no processo de medicação, em período que deve ser predeterminado, em todos os três turnos de trabalho, ou os que a instituição apresentar[31].

Posteriormente, se deseja que tenham essas palestras e cursos como círculo permanente, realizando-se uma vez a cada mês. Desta forma as palestras devem ser direcionadas, primeiramente, para o grupo de prescritores, tais como: chefes de plantão (médicos), os residentes de todas as clínicas, os acadêmicos de medicina, que permanecem por um ano em estágio no setor de emergência, e, em seguida, para os profissionais de enfermagem ${ }^{26,27}$.

\section{CONSIDERAÇÕES FINAIS}

Fica evidente que os erros com os medicamentos acontecem em função da deficiência de conhecimentos específicos, da mão-de-obra com pouca qualificação, que muitas vezes pode ser uma estratégia para diminuir custos para o hospital, além dos lapsos de memória, dos problemas com o próprio produto, com a estabilidade dos mesmos, embalagens, nomenclatura, procedimentos, prescrição, com o sistema de dispensação, distribuição e administração entre outros.

Com a revisão, viu-se que de acordo com alguns estudos recomenda-se claro para os prescritores o uso de receitas digitalizadas. Também se deve evitar o uso de abreviações, pois trazem mais de uma interpretação, um exemplo clássico de interpretação errada é quando escrevem ' $U$ ' por abreviação de unidade e quem interpreta confunde com um 'zero'. 
Os prescritores devem colocar dados sobre via de administração, forma farmacêutica e o tempo de tratamento, identificar com nome completo o paciente e leito para que não haja administração de medicamentos em outro paciente com nomes parecidos quando não idênticos. Como foi proposto, realizar programas educacionais focando na receita compreensível e nas receitas médica completas útil usar um modelo padrão de receitas, para que ocorra a diminuição na ocorrência de erros, pois um receituário em branco é muito mais suscetível a erros.

De acordo com o artigo 35, da lei 5.991 de 1973, que dispõe sobre o "controle sanitário do comércio de drogas, medicamentos, insumos farmacêuticos e correlatos", o item "a" informa que "será aviada a receita que estiver à tinta, em vernáculo por extenso e de modo legível".

No código de Ética Médica, editado pela resolução oํ 1.246/88 do Conselho Federal de Medicina, no capítulo III de responsabilidade profissional, artigo 39, a fim de que, é "proibido receitar ou atestar de forma secreta ou ilegível, assim como assinar em branco, folhas de receituários, laudos, atestados ou quaisquer outros documentos".

Em contrapartida não há consenso sobre uma receita médica ou qualquer outra escrita ilegível, pois há uma grande dúvida sobre o que é uma letra legível para os estudiosos. Determinar que as receitas médicas sejam legíveis, sem definir o que significa este termo ou qual parâmetro que define o grau de legibilidade adequada não ajuda o médico a tornar sua prescrição mais compreensível.

Não se pode descartar a interpretação das receitas, observa-se que pela grande demanda, e necessária agilidade, muitas vezes por não conseguirem interpretar a prescrição da receita médica, ainda assim, há dispensação de um medicamento. Enfermeiros, farmacêuticos ou outros profissionais da área da saúde, que se envolvem na parte de interpretação de receitas parem de tentar "traduzir" receitas ilegíveis, tomando como melhor forma de solucionar este problema, a consulta ao prescritor. 
Do ponto de vista ético, chegou-se à conclusão que ambas as áreas estão cometendo erros, seja na prescrição das receitas ou durante a interpretação das mesmas, e os sistemas que podem ser adotados pelo hospital para tornar esse trabalho menos injusto, já que o erro pode acontecer, mas pode ser minimizado. É indispensável à fiscalização de todas as prescrições médicas, podendo ser iniciada pelo próprio paciente quando a recebe em mãos, este deve ser orientado a questionar sobre o que está escrito para ser administrado com segurança.

Sugere-se que uma atitude em relação à ilegibilidade de receitas médicas deve ser realizada em caráter emergencial, para que mortes por erros de administração de medicamentos, intoxicações e danos à saúde sejam revertidas e que a qualidade de vida prevaleça sempre em foco. E pensando no dinheiro público, que as administrações deveriam ser bem controladas e fracionadas para evitar desperdício das verbas públicas, que pesam no bolso do próprio paciente, que é contribuinte.

\section{REFERÊNCIAS}

ALBARRAK, Ahmed l. et al. Assessment of legibility and completeness of handwritten and electronic prescriptions. Saudi Pharmaceutical Journal, v. 22, n. 6, p. 522-527, 2014.

ANACLETO, Tânia Azevedo et al. Medication errors and drug-dispensing systems in a hospital pharmacy. Clinics, v. 60, n. 4, p. 325-332, 2005.

ARAÚJO, Patrícia Taveira de Brito; UCHÔA, Severina Alice Costa. Avaliação da qualidade da prescrição de medicamentos de um hospital de ensino. Ciência \& Saúde Coletiva, v. 16, p. 1107-1114, 2011.

ASSIRI, Ghadah Asaad et al. What is the epidemiology of medication errors, errorrelated adverse events and risk factors for errors in adults managed in community care contexts? A systematic review of the international literature. BMJ open, v. 8, n. 5, p. e019101, 2018. 
BOTELHO, Stephanie Ferreira; MARTINS, Maria Auxiliadora Parreiras; REIS, Adriano Max Moreira. Análise de medicamentos novos registrados no Brasil na perspectiva do Sistema Único de Saúde e da carga de doença. Ciência \& Saúde Coletiva, v. 23, p. 215-228, 2018.

BYRNES, John. The value of physician leaders. Journal of Healthcare Management, v. 61 , n. 4 , p. $251-255,2016$.

CATANHEIDE, Izamara Damasceno; LISBOA, Erick Soares; SOUZA, Luis Eugenio Portela Fernandes de. Características da judicialização do acesso a medicamentos no Brasil: uma revisão sistemática. Physis: Revista de Saúde Coletiva, v. 26, p. 1335-1356, 2016.

CAVALLINI, Míriam Elias; BISSON, Marcelo Polacow. Farmácia hospitalar: um enfoque em sistemas de saúde. $2^{\mathrm{a}}$ Ed. Manole, 2010.

CLANCY, Carolyn M. Ten years after to err is human. American Journal of Medical Quality, v. 24, n. 6, p. 525-528, 2009.

CORRIGAN, Janet M. et al. (Ed.). Priority areas for national action: transforming health care quality. National Academies Press, 2003.

DALTON, Kieran; BYRNE, Stephen. Role of the pharmacist in reducing healthcare costs: current insights. Integrated pharmacy research \& practice, v. 6, p. 37, 2017.

DE MELO, Daniela Oliveira; DE CASTRO, Lia Lusitana Cardozo. Pharmacist's contribution to the promotion of access and rational use of essential medicines in SUS. Ciencia \& saude coletiva, v. 22, n. 1, p. 235-244, 2017.

DONALDSON, Molla S. et al. (Ed.). To err is human: building a safer health system. National Academies Press, 2000.

DORNAN, Tim et al. An in-depth investigation into causes of prescribing errors by foundation trainees in relation to their medical education: EQUIP study. London: General Medical Council, p. 1-215, 2009. 
HAYWARD, Rodney A. et al. Overestimating outcome rates: statistical estimation when reliability is suboptimal. Health services research, v. 42, n. 4, p. 1718-1738, 2007.

HENNEMAN, Elizabeth A. Unreported errors in the intensive care unit a case study of the way we work. Critical care nurse, v. 27, n. 5, p. 27-34, 2007.

HOFER, Timothy P.; KERR, Eve A.; HAYWARD, Rodney A. What is an error?. Effective clinical practice: ECP, v. 3, n. 6, p. 261-269, 2000.

KAUSHAL, Rainu et al. Electronic prescribing improves medication safety in community-based office practices. Journal of general internal medicine, v. 25, n. 6 , p. 530-536, 2010.

LEONCINE, Maury; ABBAS, Katia; PALADINI, Edson Pacheco. Proposta de melhorias no sistema de distribuição de medicamentos para aumento da qualidade em farmácias hospitalares. In: Anais do Congresso Brasileiro de Custos-ABC. 2007.

LIAO, T. Vivian et al. Evaluation of medication errors with implementation of electronic health record technology in the medical intensive care unit. Open Access $\mathbf{J}$ Clin Trials, v. 9, p. 31-40, 2017.

MAXWELL, Simon RJ. Rational prescribing: the principles of drug selection. Clinical Medicine, v. 16, n. 5, p. 459-464, 2016.

MOURA, Soraia Naback Chafia; DE MELO FILHA, Lucia Maria Vaz; RIBEIRO, Aline Correa. Análise de Erros nas Prescrições Médicas em uma Unidade de Pronto Atendimento do Municipio de Juiz de Fora-MG. Revista da Escola de Ciências Médicas de Volta Redonda, 2019.

PEDERSEN, Craig A.; SCHNEIDER, Philip J.; SCHECKELHOFF, Douglas J. ASHP national survey of pharmacy practice in hospital settings: dispensing and administration-2011. American Journal of Health-System Pharmacy, v. 69, n. 9, p. 768-785, 2012. 
RODZIEWICZ, Thomas L.; HIPSKIND, John E. Medical error prevention. In: StatPearls [Internet]. StatPearls Publishing, 2019.

SHAH, Kieran et al. Bar code medication administration technology: a systematic review of impact on patient safety when used with computerized prescriber order entry and automated dispensing devices. The Canadian journal of hospital pharmacy, v. 69, n. 5, p. 394, 2016.

ULMER, Cheryl et al. System Strategies to Improve Patient Safety and Error Prevention. In: Resident Duty Hours: Enhancing Sleep, Supervision, and Safety. National Academies Press (US), 2009.

WORLD HEALTH ORGANIZATION. Medication errors. 2016.

ZHANG, Jiajie; PATEL, Vimla L.; JOHNSON, Todd R. Medical error: Is the solution medical or cognitive?. Journal of the American Medical Informatics Association, v. 9, n. Supplement_6, p. S75-S77, 2002.

\section{APÊNDICE - REFERÊNCIAS DE NOTA DE RODAPÉ}

4. DORNAN, Tim et al. An in-depth investigation into causes of prescribing errors by foundation trainees in relation to their medical education: EQUIP study. London: General Medical Council, p. 1-215, 2009.

5. ALBARRAK, Ahmed I. et al. Assessment of legibility and completeness of handwritten and electronic prescriptions. Saudi Pharmaceutical Journal, v. 22, n. 6, p. 522-527, 2014.

6. ZHANG, Jiajie; PATEL, Vimla L.; JOHNSON, Todd R. Medical error: Is the solution medical or cognitive?. Journal of the American Medical Informatics Association, v. 9, n. Supplement_6, p. S75-S77, 2002.

7. HOFER, Timothy P.; KERR, Eve A.; HAYWARD, Rodney A. What is an error?. Effective clinical practice: ECP, v. 3, n. 6, p. 261-269, 2000. 
8. DONALDSON, Molla S. et al. (Ed.). To err is human: building a safer health system. National Academies Press, 2000

9. HAYWARD, Rodney A. et al. Overestimating outcome rates: statistical estimation when reliability is suboptimal. Health services research, v. 42, n. 4, p. 1718-1738, 2007.

10. CORRIGAN, Janet M. et al. (Ed.). Priority areas for national action: transforming health care quality. National Academies Press, 2003.

11. HENNEMAN, Elizabeth A. Unreported errors in the intensive care unit a case study of the way we work. Critical care nurse, v. 27, n. 5, p. 27-34, 2007.

12. ULMER, Cheryl et al. System Strategies to Improve Patient Safety and Error Prevention. In: Resident Duty Hours: Enhancing Sleep, Supervision, and Safety. National Academies Press (US), 2009.

13. BYRNES, John. The value of physician leaders. Journal of Healthcare Management, v. 61, n. 4, p. 251-255, 2016.

14. MAXWELL, Simon RJ. Rational prescribing: the principles of drug selection. Clinical Medicine, v. 16, n. 5, p. 459-464, 2016.

15. SANT'ANA, João Mauricio Brambati et al. Racionalidade terapêutica: elementos médico-sanitários nas demandas judiciais de medicamentos. Revista de Saúde Pública, v. 45, p. 714-721, 2011.

16. CATANHEIDE, Izamara Damasceno; LISBOA, Erick Soares; SOUZA, Luis Eugenio Portela Fernandes de. Características da judicialização do acesso a medicamentos no Brasil: uma revisão sistemática. Physis: Revista de Saúde Coletiva, v. 26, p. 1335-1356, 2016.

17. ARAÚJO, Patrícia Taveira de Brito; UCHÔA, Severina Alice Costa. Avaliação da qualidade da prescrição de medicamentos de um hospital de ensino. Ciência \& Saúde Coletiva, v. 16, p. 1107-1114, 2011. 
18. ASSIRI, Ghadah Asaad et al. What is the epidemiology of medication errors, errorrelated adverse events and risk factors for errors in adults managed in community care contexts? A systematic review of the international literature. BMJ open, v. 8, n. 5, p. e019101, 2018.

19. ANACLETO, Tânia Azevedo et al. Medication errors and drug-dispensing systems in a hospital pharmacy. Clinics, v. 60, n. 4, p. 325-332, 2005.

20. WORLD HEALTH ORGANIZATION. Medication Errors: Technical Series on Safer Primary Care, 2016.

21. DE MELO, Daniela Oliveira; DE CASTRO, Lia Lusitana Cardozo. Pharmacist's contribution to the promotion of access and rational use of essential medicines in SUS. Ciencia \& saude coletiva, v. 22, n. 1, p. 235-244, 2017.

22. LEONCINE, Maury; ABBAS, Katia; PALADINI, Edson Pacheco. Proposta de melhorias no sistema de distribuição de medicamentos para aumento da qualidade em farmácias hospitalares. In: Anais do Congresso Brasileiro de Custos-ABC. 2007.

23. SHAH, Kieran et al. Bar code medication administration technology: a systematic review of impact on patient safety when used with computerized prescriber order entry and automated dispensing devices. The Canadian journal of hospital pharmacy, v. 69, n. 5 , p. 394, 2016.

24. BOTELHO, Stephanie Ferreira; MARTINS, Maria Auxiliadora Parreiras; REIS, Adriano Max Moreira. Análise de medicamentos novos registrados no Brasil na perspectiva do Sistema Único de Saúde e da carga de doença. Ciência \& Saúde Coletiva, v. 23, p. 215-228, 2018.

25. KAUSHAL, Rainu et al. Electronic prescribing improves medication safety in community-based office practices. Journal of general internal medicine, v. 25, n. 6, p. 530-536, 2010. 
26. CAVALLINI, Míriam Elias; BISSON, Marcelo Polacow. Farmácia hospitalar: um enfoque em sistemas de saúde. 2aㅡ Ed. Manole, 2010.

27. LIAO, T. Vivian et al. Evaluation of medication errors with implementation of electronic health record technology in the medical intensive care unit. Open Access $\mathrm{J}$ Clin Trials, v. 9, p. 31-40, 2017.

28. PEDERSEN, Craig A.; SCHNEIDER, Philip J.; SCHECKELHOFF, Douglas J. ASHP national survey of pharmacy practice in hospital settings: dispensing and administration-2011. American Journal of Health-System Pharmacy, v. 69, n. 9, p. 768-785, 2012.

29. DALTON, Kieran; BYRNE, Stephen. Role of the pharmacist in reducing healthcare costs: current insights. Integrated pharmacy research \& practice, v. 6, p. 37, 2017.

30. LESAR, Timothy S. et al. The VHA New England Medication Error Prevention Initiative as a model for long-term improvement collaboratives. The Joint Commission Journal on Quality and Patient Safety, v. 33, n. 2, p. 73-82, 2007.

31. RODZIEWICZ, Thomas L.; HIPSKIND, John E. Medical error prevention. In: StatPearls [Internet]. StatPearls Publishing, 2019.

Enviado: Janeiro, 2020.

Aprovado: Janeiro, 2020. 